\title{
LA-UR-97- 3260
}
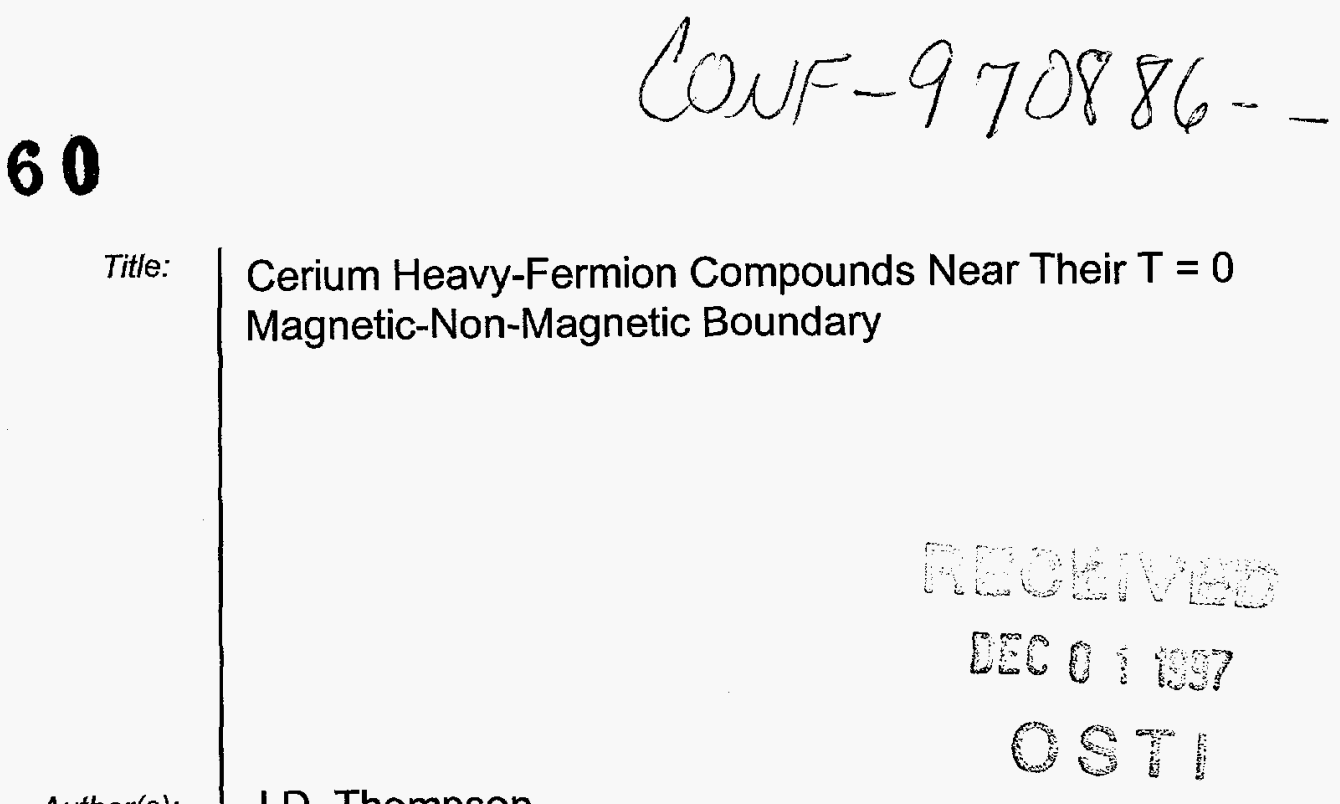

Submitted to:

\section{J.D. Thompson}

T. Graf

M.F. Hundley

R. Movshovich

J.L. Sarrao

Y. Uwatoko

Z. Fisk

R. Fisher

N. Phillips

The Review of High Pressure Science and Technolgoy

Kyoto Japan

Aug. 25, 1997

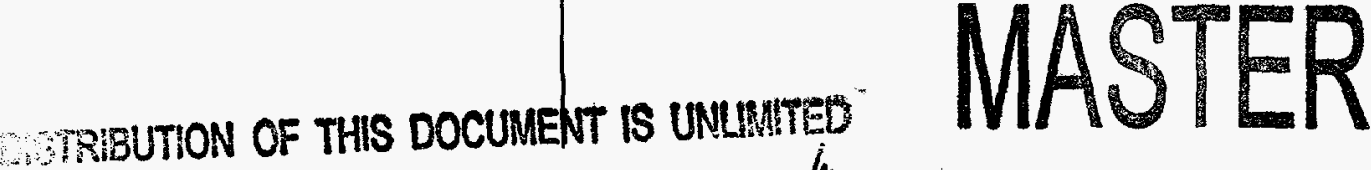

\section{Los Alamos}

NATIONAL LABORATOR

Los Alamos National Laboratory, an affirmative action/equal opportunity employer, is operated by the University of California for the U.S. Department of Energy under contract W-7405-ENG-36. By acceptance of this article, the publisher recognizes that the U.S. Government retains a nonexclusive, royalty-free license to publish or reproduce the published form of this contribution, or to allow others to do so, for U.S. Government purposes. The Los Alamos National Laboratory requests that the publisher identify this article as work performed under the auspices of the U.S. Department of Energy. 


\section{DISCLAIMER}

This report was prepared as an account of work sponsored by an agency of the United States Government. Neither the United States Government nor any agency thereof, nor any of their employees, makes any warranty, express or implied, or assumes any legal liability or responsibility for the accuracy, completeness, or usefulness of any information, apparatus, product, or process disclosed, or represents that its use would not infringe privately owned rights. Reference herein to any specific commercial product, process, or service by trade name, trademark, manufacturer, or otherwise does not necessarily constitute or imply its endorsement, recommendation, or favoring by the United States Government or any agency thereof. The views and opinions of authors expressed herein do not necessarily state or reflect those of the United States Government or any agency thereof. 


\section{DESCAMER}

Portions of this docoment miy be illegible in electronic image products. Images are produced from the best available original docomentert 


\title{
Cerium Heavy-Fermion Compounds Near Their $\mathbf{T}=0$ Magnetic-Non- Magnetic Boundary
}

\author{
J.D. Thompson, T. Graf*, M.F. Hundley, Y. Uwatoko ${ }^{\dagger}$, R. Movshovich, J.L. Sarrao, Z. \\ Fisk $^{\ddagger}$, R.A. Fisher**, and N.E. Phillips** \\ Los Alamos National Laboratory, Los Alamos, NM 87545 \\ * ETH Hönggerberg, CH-8093 Zurich, Switzerland \\ $\dagger^{\top}$ Faculty of Science, Saitama University, Urawa, Japan \\ $\ddagger$ NHMFL, Florida State University, Tallahassee, FL 32310 \\ ** LBNL and Department of Chemistry University of California, \\ Berkeley, CA 94270
}

Measurements of the temperature-dependent specific heat and thermal expansion coefficient near a $\mathrm{T}=0$ magnetic-non-magnetic boundary, accessed in $\mathrm{CeRh}_{2} \mathrm{Si}_{2}$ by application of pressure and in $\mathrm{CeRh}_{2-\mathrm{x}} \mathrm{Ru}_{\mathrm{x}} \mathrm{Si}_{2}$ at ambient pressure by chemical substitution, emphasize the role of disorder in producing non-Fermi-liquid behavior. Interestingly, superconductivity also develops near this boundary in some crystallographically-ordered Ce-based heavy-fermion compounds. [CeRh ${ }_{2-x} \mathrm{Ru}_{\mathrm{x}} \mathrm{Si}_{2}$, specific heat, thermal expansion, susceptibility, non-Fermi-liquid] 


\section{Introduction}

The study of correlated-electron materials near a zero-temperature magnetic-nonmagnetic boundary is intrinsically interesting both experimentally and theoretically. Within the past few years, special attention has been drawn to Kondo-lattice/heavyfermion materials in this limit. Observation of logarithmic or unusual power-law temperature dependences of thermodynamic and transport properties have suggested a non-Fermi-liquid (NFL) groundstate in several Ce- and U-based Kondo-lattice systems in which a $\mathrm{T}=0$ magnetic-non-magnetic boundary has been accessed by application of pressure or by chemical substitutions.[1] These findings have led to several theoretical proposals for the origin of NFL behavior, including quantum-critical fluctuations [2], a multichannel Kondo effect [3], local spin fluctuations near an antiferromagnetic instability [4], and a distribution of Kondo temperatures introduced by crystallographic disorder with associated hybridization disorder [5,6]. Attempts to describe NFL behavior by these models have met with varying degrees of success, and no consensus has emerged for the origin of these effects. However, many of the materials in which NFL behavior has been observed contain some form of disorder, either produced by chemical substitution on for ligand sites or that is intrinsic to the material's crystal structure.

Comparably interesting has been the observation of superconductivity that develops in some Ce-based Kondo-lattice systems as their magnetic-non-magnetic boundary is driven toward $\mathrm{T}=0$ by application of pressure. Materials (and critical pressures $\mathrm{P}_{\mathrm{c}}$ ) where this phenomenon has been found include: $\mathrm{CeCu}_{2} \mathrm{Ge}_{2}(\sim 77 \mathrm{kbar})$ [7], $\mathrm{CePd}_{2} \mathrm{Si}_{2}$ ( $\sim 27 \mathrm{kbar}$ ) [8], $\mathrm{CeRh}_{2} \mathrm{Si}_{2}$ ( $\left.\sim 9 \mathrm{kbar}\right)$ [9], $\mathrm{CeNi}_{2} \mathrm{Ge}_{2}(\sim 15 \mathrm{kbar})$ [10] and possibly $\mathrm{CeCu}_{2}$

( $\sim 60 \mathrm{kbar}$ ) [11]. This pressure-induced superconductivity is not always reproducible but 
appears to depend sensitively on sample "quality". Although what precisely is meant by "quality" remains to be determined, there seems to be a correlation between lower residual resistivity and the appearance of superconductivity [12]. Variations in residual resistivity could come from crystallographic imperfections and/or fluctuations associated with proximity to a magnetic transition, but, again, this needs to be established. In any event, the development of superconductivity near a pressure-induced magnetic-nonmagnetic boundary suggests that Cooper pairing may be mediated by some form of magnetic fluctuations and, in analogy to other heavy-fermion superconductors, that the superconductivity is unconventional. Notably, superconductivity has not been found in those materials whose $\mathrm{T}=0$ magnetic-non-magnetic boundary has been accessed by partial substitutions on either the $f$ or ligand sites.

$\mathrm{CeRh}_{2} \mathrm{Si}_{2}$ is a Kondo-lattice system whose Neél temperature $\left(\mathrm{T}_{\mathrm{N}} \approx 35 \mathrm{~K}\right)$ can be driven to zero at $\mathrm{P}_{\mathrm{c}} \approx 9 \mathrm{kbar}[13]$ or at atmospheric pressure by substituting $\mathrm{Ru}$ for

- $\mathrm{Rh}[14]$. In the following, we compare the temperature dependence of physical properties of these materials near their respective $\mathrm{T}=0$ magnetic-non-magnetic boundaries.

\section{Experimental}

Polycrystalline samples of $\mathrm{CeRh}_{2} \mathrm{Si}_{2}$ and $\mathrm{CeRh}_{2-\mathrm{x}} \mathrm{Ru}_{\mathrm{x}} \mathrm{Si}_{2}(\mathrm{x} \approx 1)$ were prepared by arc melting in an inert atmosphere. In the case of $\mathrm{CeRh}_{0.96} \mathrm{Ru}_{1.04} \mathrm{Si}_{2}$, sufficient grain growth on the surface of the arc-melted button allowed a small single crystal to be mechanically removed for magnetic susceptibility measurements. Powder x-ray diffraction on all samples showed them to be single-phase with the $\mathrm{ThCr}_{2} \mathrm{Si}_{2}$ structure. However, specific heat measurements on Ru-doped samples revealed weak anomalies near 5 and $11 \mathrm{~K}$ for $\mathrm{x}=1.04$ and 1.0 , respectively. In view of the magnetic phase diagram for $C e \mathrm{Rh}_{2-\mathrm{x}} \mathrm{Ru}_{\mathrm{x}} \mathrm{Si}_{2}$ 
[14], these are consistent with the presence of less than 1 mole-percent of slightly Rudeficient second phase. Specific heat (thermal expansion) measurements on $\mathrm{CeRh}_{2} \mathrm{Si}_{2}$ under pressure were performed in a $\mathrm{Be}-\mathrm{Cu}$ clamp with $\mathrm{AgCl}$ (Fluorinert) as the pressuretransmitting medium. Thermal expansion measurements at ambient pressure were obtained with a high resolution $\left(\Delta \ell / \ell \approx 10^{-8}\right)$ capacitance dilatometer and those as a function of pressure with a strain-gauge technique [15].

\section{Results}

Figure 1 shows the magnetic specific heat $\mathrm{C}_{\mathrm{m}}$ divided by temperature for $\mathrm{CeRh}_{2} \mathrm{Si}_{2}$ at pressures below and above the critical pressure $P_{c}$ required to suppress its Neél temperature to zero. For pressures sufficiently close to $P_{c}$, quantum-critical fluctuations should produce a logarithmic or stronger divergence of $C_{m} / T$ at low temperatures $[2,4]$; however, these data show no significant temperature dependence below a few Kelvins. It is possible that data have not been acquired sufficiently near the $\mathrm{T}=0$ magnetic-nonmagnetic boundary to observe NFL behavior. Taking $\left|P / P_{c}-1\right|=\Delta$ as a measure of nearness to the quantum-critical point, then $\Delta \geq 0.2$ for the specific data in Fig. 1. For $P / P_{c}=0.79, \mathrm{~T}_{\mathrm{N}}(0.79)$ is approximately $25 \mathrm{~K}$ and thus $T_{N}(0.79) / T_{N}(0) \approx 0.7 .[13,16]$ By this measure, data at $\mathrm{P} / \mathrm{P}_{\mathrm{c}}=0.79$ are relatively far from a critical point, and the absence of NFL behavior is not surprising.

This issue of proximity to the $\mathrm{T}=0$ transition has been addressed more carefully by thermal expansion measurements. Commonly, properties of Kondo-lattice/heavyfermion materials are analyzed assuming that the electronic contribution to their free energy scales with a single volume-dependent characteristic energy scale, often associated with the Kondo temperature $T_{\mathrm{K}}$.[17] With this assumption, the correlated- 
electron contribution to the volume-thermal expansion coefficient $\beta$ is related to the specific heat by $\beta=\Gamma \kappa C_{m} / V$, where $\kappa$ is the compressibility, $V$ is the volume and $\Gamma$ is the electronic Grüneisen parameter. If $\Gamma$ and $\kappa$ are only weakly affected by quantumcritical fluctuations, then $\beta$ should exhibit approximately the same temperature dependence as $\mathrm{C}_{\mathrm{m}}$.

Figure 2 shows $\beta / T$ versus temperature on a logarithmic scale for $\mathrm{CeRh}_{2} \mathrm{Si}_{2}$ at pressures relatively nearer to $P_{c}$. For $P / P_{c}=0.72$ and 0.89 , large negative anomalies appear at $\mathrm{T}_{\mathrm{N}}(\mathrm{P})$. This behavior is expected on the basis of Ehrenfest's relationship and the observed steep decrease in $T_{N}$ as $P$ approaches $P_{c} \cdot[13,16]$ For $T>T_{N}(P), \beta / T$ is positive and close to the curve obtained at $P / P_{c}=1.09$. Rather interesting are data obtained closest to the critical point. Here, $\beta / \mathrm{T}$ begins to decrease below about $10 \mathrm{~K}$ and appears to diverge approximately logarithmically with decreasing temperatures. If this divergence were due to NFL behavior, it would require that the Grüneisen parameter be negative in this regime. Except for possibly $\mathrm{CeAl}_{3}$ at very low temperatures, the electronic Grüneisen parameter of Ce-based heavy-fermion materials is positive.[17] Consequently, a negative $\Gamma$ for $\mathrm{CeRh}_{2} \mathrm{Si}_{2}$ would be a very unusual situation and, additionally, appears to conflict with results, discussed below, for $C e R h_{2-x} \mathrm{Ru}_{\mathrm{x}} \mathrm{Si}_{2}$.

An alternative interpretation of this curve for $P / P_{c}=0.97$ is that the decrease in $\beta / T$ beginning near $10 \mathrm{~K}$ reflects the approach to a magnetic transition below $3 \mathrm{~K}$. In this case, the transition would have to be exceeding broad, with a width greater than $7 \mathrm{~K}$, which is not the case at lower pressures. Near $\mathrm{P}_{\mathrm{c}}, \partial \ln T_{N} / \partial P \approx-0.55 \mathrm{~K} / \mathrm{kbar}$ for $\mathrm{CeRh}_{2} \mathrm{Si}_{2}$. For the transition to extend from roughly 10 to $3 \mathrm{~K}$ would imply a pressure gradient $\Delta \mathrm{P} \approx 1.2 \mathrm{kbar}$ in the pressure clamp, if all the broadening were due to non- 
hydrostaticity. Careful measurements in this clamp on highly stress-dependent superconducting transitions indicated that the maximum pressure gradient is less than $0.01 \mathrm{P}$, or equivalently $0.1 \mathrm{kbar}$ at a pressure of $10 \mathrm{kbar}\left(\approx \mathrm{P}_{\mathrm{c}}\right)$. [18] Even if the relative change in $T_{N}$ near $P_{c}$ were underestimated by a factor of two, it seems unlikely that a pressure gradient in the clamp could account for the unusual temperature dependence of $\beta / T$ at $P / P_{c}=0.97$. Presently we have no clear understanding of this result, but additional measurements, e.g. thermal expansion to lower temperatures and specific heat at pressures closer to $P_{c}$, should be informative.

It is interesting to compare specific heat and thermal expansion measurements on $\mathrm{CeRh}_{2} \mathrm{Si}_{2}$ under pressure to those obtained at ambient pressure when the $\mathrm{CeRh}_{2} \mathrm{Si}_{2}$ is alloyed with $\mathrm{Ru}$. Substituting $\mathrm{Ru}$ for $\mathrm{Rh}$ in $\mathrm{CeRh} \mathrm{h}_{2-\mathrm{x}} \mathrm{Ru}_{\mathrm{x}} \mathrm{Si}_{2}$ initially depresses $\mathrm{T}_{\mathrm{N}}$ rapidly to a plateau where, for $0.2<\mathrm{x}<0.8, \mathrm{~T}_{\mathrm{N}}$ is about $11 \mathrm{~K}$. With additional $\mathrm{Ru}, \mathrm{T}_{\mathrm{N}}$ drops abruptly to zero at a critical $\mathrm{Ru}$ concentration $\mathrm{x}_{\mathrm{c}} \approx 0.95$.[14] Magnetic susceptibility $\chi$ and specific heat measurements on a polycrystalline sample with $\mathrm{x} \approx 1.0$ show a NFLlike logarithmic increase in $\chi$ and $\mathrm{C}_{\mathrm{m}} / \mathrm{T}$ over an interval spanning more than one decade in temperature above 1 K.[16] However, this NFL behavior does not persist in the ground state of $\mathrm{CeRhRuSi}_{2} ;$ instead, $\mathrm{C}_{\mathrm{m}} / \mathrm{T}$ approaches a large, nearly temperature-independent value as $\mathbf{T}$ goes to zero, typical of a strongly correlated Fermi liquid. The temperature dependence and magnitude of both $\chi$ and $\mathrm{C}_{\mathrm{m}}$ could be interpreted consistently assuming that crystallographic disorder introduced by Ru substitution produced a moderately narrow Gaussian distribution of Kondo temperatures around a mean value of about $30 \mathrm{~K} .[16]$ 
Figure 3 compares the temperature dependence of $C_{m} / T$ and $\beta / T$ for a polycrystalline sample with $\mathrm{x} \approx 1.0$. (We have assumed that the volume-thermal expansion coefficient is equal to three times the measured linear coefficient, even though the crystal structure is tetragonal. This assumption should not strongly influence the temperature dependence shown, but probably does affect the quantitative magnitude of $\beta$.) As expected from the thermodynamic relationship between $\beta$ and $C_{m}$ given earlier, $\beta / T$ increases logarithmically with decreasing temperature over essentially the same temperature interval where $C_{m} / T \propto-\ell n T$. The ratio $\beta / C_{m} \propto \Gamma$ is weakly temperature dependent for 1 $<\mathrm{T}<10 \mathrm{~K}$, which can be accounted for [19] semiquantitatively if the distribution of Kondo temperatures also influences $\Gamma$. The divergence of $\beta / T$ for $C e \mathrm{Rh}_{2-x} \mathrm{Ru}_{\mathrm{x}} \mathrm{Si}_{2}$ near its $\mathrm{T}=0$ magnetic-non-magnetic boundary contrasts strongly with results for $\mathrm{CeRh}_{2} \mathrm{Si}_{2}$.

Because the Kondo effect arises from hybridization of conduction electrons with a local f-moment, spatial variations in the hybridization matrix element, and correspondingly in $\mathrm{T}_{\mathrm{K}}$, should produce a NFL-temperature dependence in potentially anisotropic physical properties of a disordered system, such as the magnetic susceptibility in $\mathrm{CeRh}_{2-\mathrm{x}} \mathrm{Ru}_{\mathrm{x}} \mathrm{Si}_{2}$. Figure 4 gives the temperature dependence of $\chi$ for a magnetic field applied parallel and perpendicular to the c-axis of a small crystal of $\mathrm{CeRh} .{ }_{96} \mathrm{Ru}_{1.04} \mathrm{Si}_{2}$. The specific heat of this material is identical to that shown in Fig. 3(a) for CeRhRuSi 2 . Although there is large anisotropy in the magnitude of $\chi$, both orientations show $-\ell n T$ dependence in the range $5 \leq \mathrm{T} \leq 50 \mathrm{~K}$. The weak upturn at low temperatures is sample dependent, suggesting that it arises from trace impurities. A polycrystalline average of these data agrees well with that found earlier [16] on polycrystal CeRhRuSi $i_{2}$. The somewhat larger low-temperature value of $\chi_{\text {ave }}$, compared to $\mathrm{CeRhRuSi}{ }_{2}$, improves 
agreement [16] between the mean Kondo temperatures derived by fitting the Kondodisorder model [5] to $\chi$ and $\mathrm{C}_{\mathrm{m}} / \mathrm{T}$.

\section{Discussion}

As discussed elsewhere in these proceedings [20], there is growing evidence for NFL behavior in Ce-based materials near their $\mathrm{T}=0$ magnetic-non-magnetic boundary. However, except for indications of departure from a Fermi-liquid-like quadratic temperature dependence of the low-temperature resistivity $\rho$ in $\mathrm{CePd}_{2} \mathrm{Si}_{2}[8]$ and $\mathrm{CeNi}_{2} \mathrm{Ge}_{2}[10,21]$, there remains no unequivocal evidence for NFL behavior in crystallographically ordered Ce-compounds whose $\mathrm{T}=0$ boundary has been accessed by pressure. $\mathrm{CeRh}_{2} \mathrm{Si}_{2}$ is no exception. The NFL-like $\rho \propto \mathrm{AT}^{\mathrm{n}}(1.1<\mathrm{n}<1.6)$ found in $\mathrm{CePd}_{2} \mathrm{Si}_{2}$ and $\mathrm{CeNi}_{2} \mathrm{Ge}_{2}$ also appears to depend sensitively on the magnitude of the residual resistivity $\rho_{\mathrm{o}}$ and/or crystallographic direction. For example, a $\mathrm{CePd}_{2} \mathrm{Si}_{2}$ sample with a modestly large $\rho_{0} \approx 20 \mu \Omega \mathrm{cm}$ gives $\rho \propto \mathrm{AT}^{2}$ near the $\mathrm{T}=0$ boundary [12] in contrast to $\rho \propto \mathrm{T}^{1.2}$ in a sample with $\rho_{0} \approx 5 \mu \Omega \mathrm{cm}$ [8], and the c-axis resistivity of $\mathrm{CeNi}_{2} \mathrm{Ge}_{2}$ increases at $\mathrm{T}^{2}$ at ambient and high pressures [22] but reports [10,21] on polycrystalline samples find $\rho \propto T^{-1.6}$. What relationship, if any, there is between the existence of NFL-like behavior in these (and isostructural) compounds and the appearance or absence of superconductivity near the $T=0$ magnetic-non-magnetic boundary is an outstanding question whose answer may provide a fundamental clue to the origin of superconductivity in heavy-fermion compounds in general.

In contrast to well-ordered compounds, there is ample evidence for NFL-like thermodynamic and transport properties in systems whose $\mathrm{T}=0$ magnetic-non-magnetic 
boundary has been reached by chemical substitutions. The absence of superconductivity in these materials is not surprising because the chemical (and associated hybridization) disorder introduces both elastic and inelastic scattering that would be detrimental to superconductivity, especially if it were unconventional. Although no consensus has emerged for a common interpretation of the origin of NFL behavior in these disordered systems, there is mounting experimental evidence that disorder should not be neglected in any eventual model of NFL behavior in these materials. Precisely what role disorder plays remains to be established unambiguously. It is clear, however, from a number of studies that NFL effects can be found in disordered materials that are relatively far from a $\mathrm{T}=0$ boundary by virtually any measure of nearness to this boundary. An interesting case is $\mathrm{CeRh}_{2-\mathrm{x}} \mathrm{Ru}_{\mathrm{x}} \mathrm{Si}_{2}$ in which $\mathrm{C}_{\mathrm{m}} / \mathrm{T} \propto-\ln T$ even for $\mathrm{x}=1.4$.[23] This would suggest that disorder may be more important than proximity to a quantum critical point for producing NFL behavior in these materials.

\section{Summary}

The contrasting behaviors of $\mathrm{CeRh}_{2} \mathrm{Si}_{2}$ and $\mathrm{CeRh}_{2-\mathrm{x}} \mathrm{Ru}_{\mathrm{x}} \mathrm{Si}_{2}$ near their respective $\mathrm{T}=0$ magnetic-non-magnetic boundary are striking. Even when measurements are performed relatively near the critical point in $\mathrm{CeRh}_{2} \mathrm{Si}_{2}\left(\mathrm{P} / \mathrm{P}_{\mathrm{c}}=0.97\right.$ and 1.09), there is no clear evidence for NFL behavior that might be expected from quantum-critical fluctuations. Extending these thermodynamic measurements to lower temperatures and to pressures yet closer to $P_{c}$ should help clarify conditions under which NFL effects appear in wellordered compounds. On the other hand, chemical and attendant hybridization disorder in $\mathrm{CeRh}_{2-\mathrm{x}} \mathrm{Ru}_{\mathrm{x}} \mathrm{Si}_{2}$ produces $\mathrm{NFL}$-like behavior even relatively far from a quantum-phase 
transition. Pressure studies on well-characterized, preferably single-crystal, samples will be necessary to understand the relationship between NFL features and the appearance of superconductivity near a $\mathrm{T}=0$ magnetic-non-magnetic boundary in isostructural $\mathrm{CeM}_{2} \mathrm{X}_{2}$ compounds.

\section{Acknowledgement}

Work at Los Alamos was performed under the auspices of the U.S. Department of Energy and work at Lawrence Berkeley Laboratory was supported by the Director, Office of Basic Energy Research, Division of Materials Sciences of the U.S. DOE under Contract No. DE-AC03-765F0098. 
Figure Captions

Fig. 1 Magnetic specific heat $\mathrm{C}_{\mathrm{m}}$ divided by temperature versus temperature on a logarithmic scale for $\mathrm{CeRh}_{2} \mathrm{Si}_{2}$ at various pressures $\mathrm{P}$ normalized by the critical pressure $\mathrm{P}_{\mathrm{c}}$. The lattice specific heat of $\mathrm{CeRh}_{2} \mathrm{Si}_{2}$ was approximated by that of $\mathrm{LaRhRuSi}_{2}$ and subtracted from the total specific heat to obtain $\mathrm{C}_{\mathrm{m}}$.

Fig. 2 Volume-thermal expansion coefficient $\beta$ divided by temperature as a function of temperature on a logarithmic scale for $\mathrm{CeRh}_{2} \mathrm{Si}_{2}$ at various pressure $\mathrm{P}$ normalized by the critical pressure $P_{c}$. We have assumed that $\beta$ is equal to three times the measured linearexpansion coefficient even though the material is tetragonal. Note the absence of a divergence in $\beta / T$ for $P / P_{c}=1.09$.

Fig. 3(a) Magnetic specific heat divided by temperature versus temperature on a logarithmic scale for $\mathrm{CeRhRuSi}_{2}$ at atmospheric pressure. (b) Volume-thermal expansion coefficient divided by temperature versus temperature on a logarithmic scale. Solid lines are guides to the eye. Note that $C_{m} / T$ and $\beta / T$ both diverge logarithmically over approximately the same temperature interval.

Fig. 4 Magnetic susceptibility of $\mathrm{CeRh}_{.96} \mathrm{Ru}_{1.04} \mathrm{Si}_{2}$ for a $1 \mathrm{kOe}$ field applied parallel (circles) and perpendicular (triangles) to its c-axis. Connected diamonds are a polycrystalline average of the two other data sets. All three sets of data are proportional to $-\ln \mathrm{T}$ over the interval $\sim 5 \leq \mathrm{T} \leq 50 \mathrm{~K}$. 


\section{References}

1. See, for example, Proceedings of the Workshop on Non-Fermi-Liquid Behavior in Solids, J. Phys.: Condens. Matter $\underline{8}(1996)$.

2. M.A. Continentino, Phys. Rep. $\underline{239}, 179$ (1994).

3. D.L. Cox, Phys. Rev. Lett. $\underline{59}, 1240$ (1987); A. M. Tsvelich, J. Phys.: Condens. Matter $\underline{2}$, 2833 (1990).

4. A. J. Millis, Phys. Rev. B 4 8, 7183 (1993); U. Züliche and A.J. Millis, Phys. Rev. B $\underline{51}, 8996$ (1995); T. Moriya and T. Takimoto, J. Phys. Soc. Jpn. 64, 960 (1995).

5. O.O. Bernal, D.E. MacLaughlin, H.G. Lukefar, and B. Andraka, Phys. Rev. Lett. 75 , 2023 (1995).

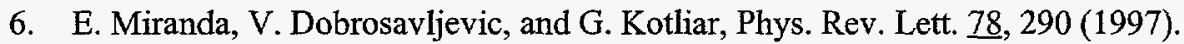

7. D. Jaccard, K. Behnia, and J. Sierro, Phys. Lett. A $\underline{163}, 475$ (1992).

8. F.M. Grosche, S.R. Julian, N.D. Mathur, and G.G. Lonzarich, Physica B 223 \& 224, 50, (1996).

9. R. Movshovich et al., Physica B 223 \& 224, 126 (1996).

10. S.J.S. Lister et al., Z. Physik $\underline{103}$, 263, (1997).

11. E. Vargoz, P. Link, and D. Jaccard, Physica B 230-232, 182 (1997).

12. P. Link, D. Jaccard, and P. Lejay, Physica B $\underline{223 ~ \& ~ 224, ~} 303$ (1996).

13. J.D. Thompson, R. D. Parks, and H.A. Borges, J. Magn. Magn. Mater. 54-57, 281 (1985).

14. S. Kawarazaki et al., Physica B 206-207, 298 (1995).

15. Y. Uwatoko and G. Oomi, J. High Pressure Inst. Jpn. 29, 609 (1991) (in Japanese).

16. T. Graf et al., Phys. Rev. Lett. $\underline{78}, 3769$ (1997).

17. See, for example, J.D. Thompson and J.M. Lawrence, in Handbook on the Physics and Chemistry of Rare Earths, vol. 19 - Lanthanides/Actinides: Physics - II, eds. K.A. Gschneider, L. Eyring, G.H. Lander and G. R. Choppin (Elsevier, Amsterdam, 1994) p. 383.

18. J.D. Thompson, unpublished.

19. J.D. Thompson, M.F. Hundley, and J.L. Sarrao, J. Alloys and Compounds, submitted.

20 R.K. Haselwimmer et al., these proceedings; G. Oomi et al., these proceedings; G. Sparn et al., these proceedings.

21. F. Steglich et al., J. Phys.: Condens. Matter $\underline{8}, 9909$ (1996). 
22. F. Fukuhara et al., Physica B 230-232, 198 (1997).

23. T. Taniguchi, Y. Tabata, H. Tanabe, and Y. Miyako, Physica B 230-232, 123 (1997). 


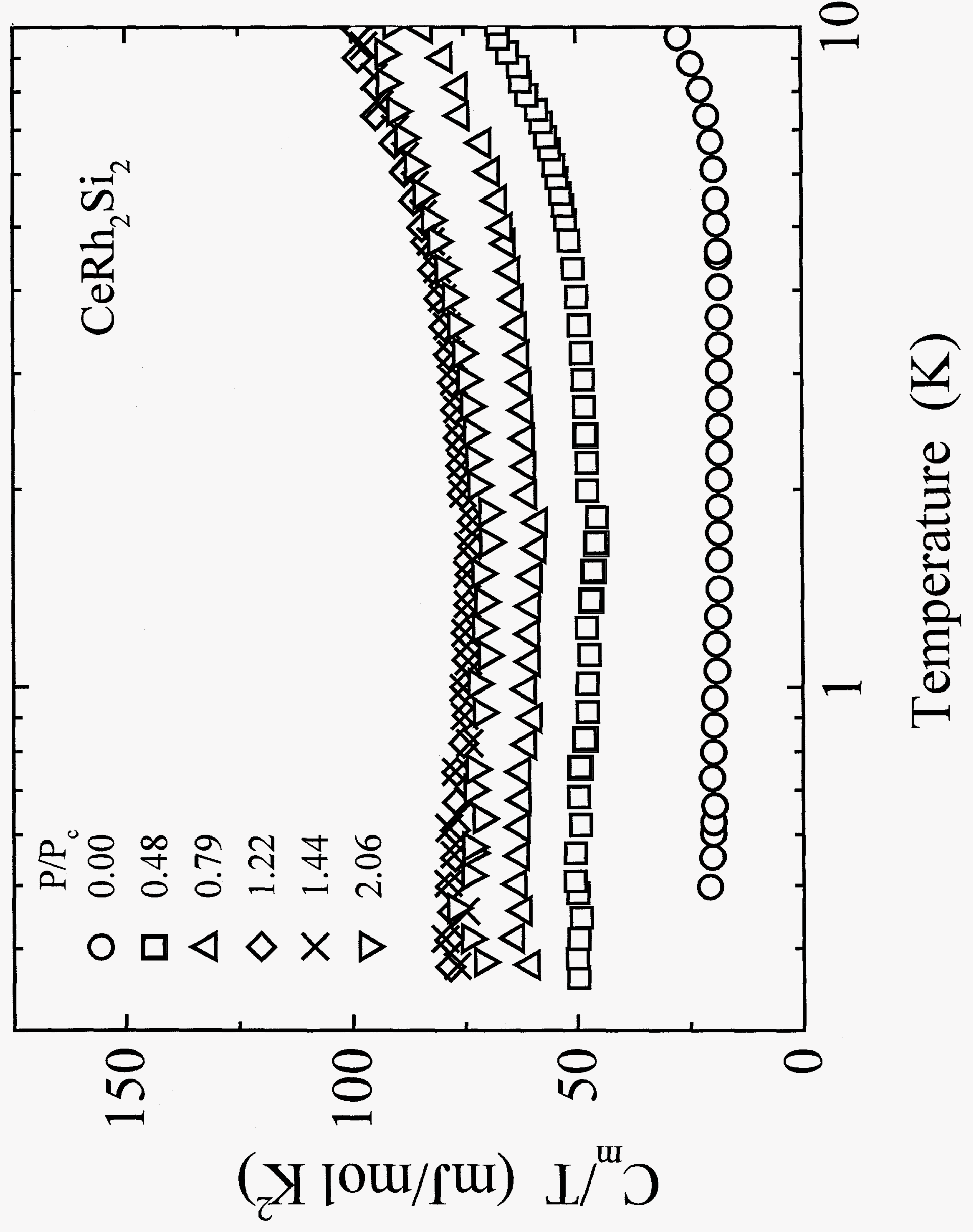




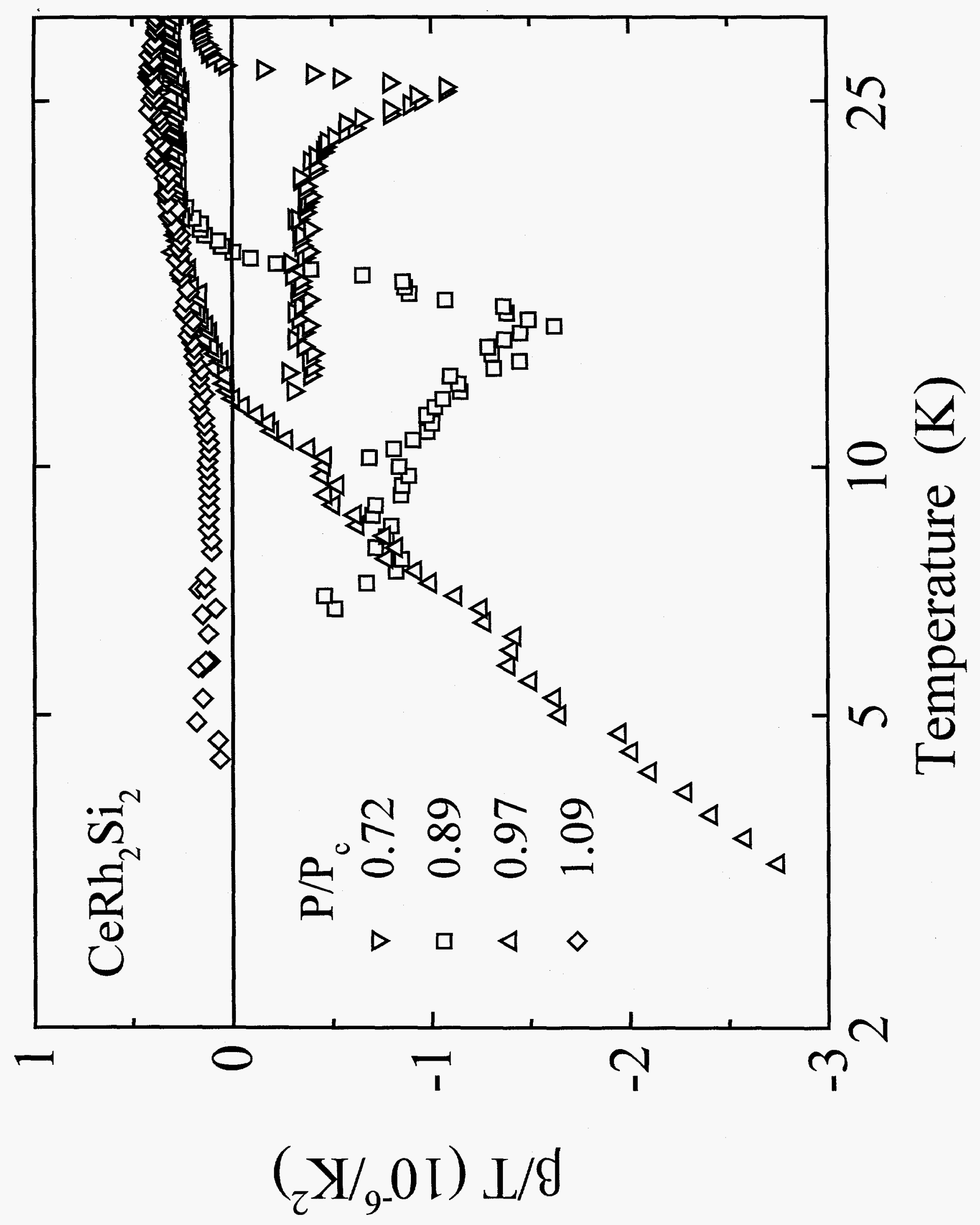



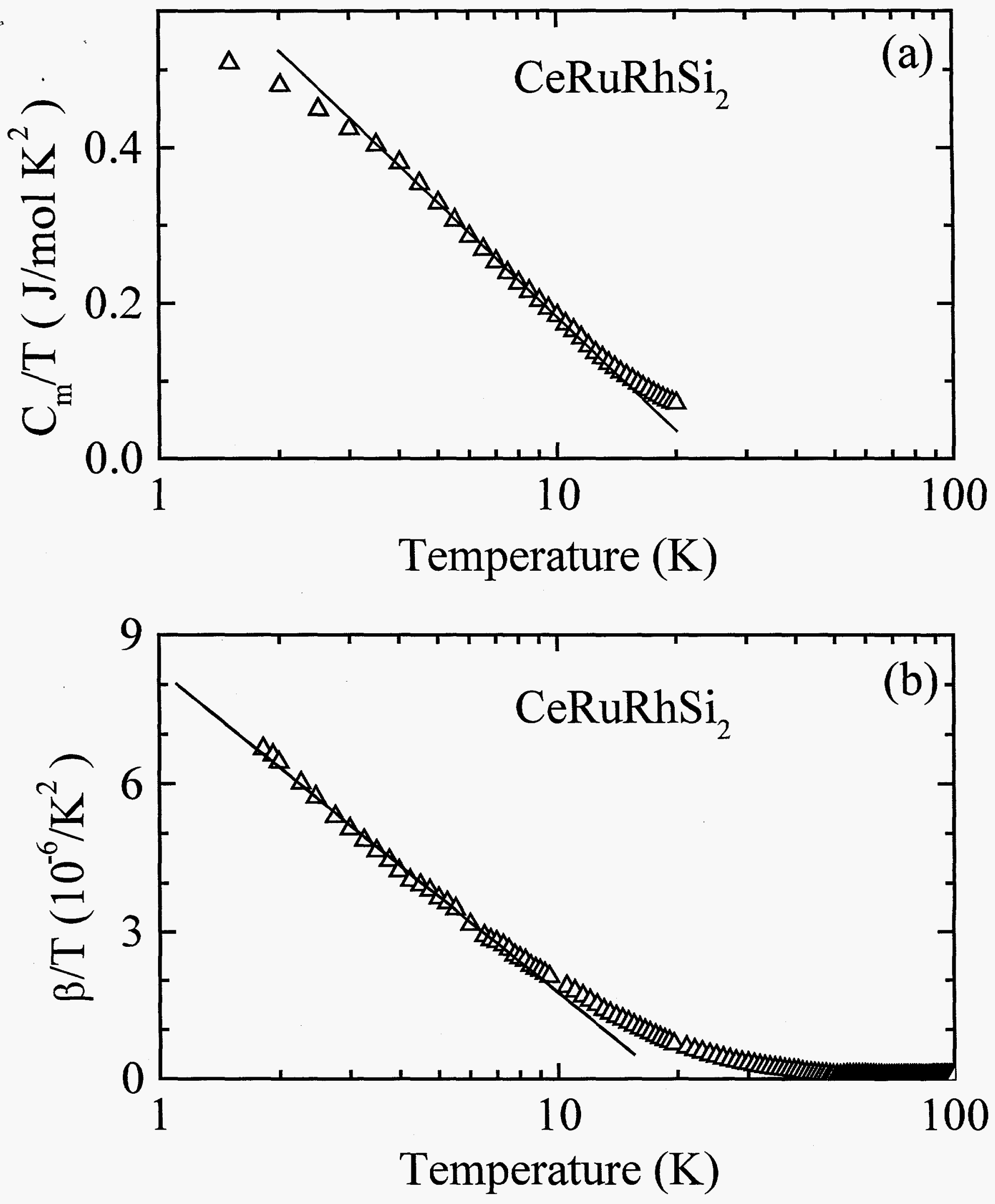


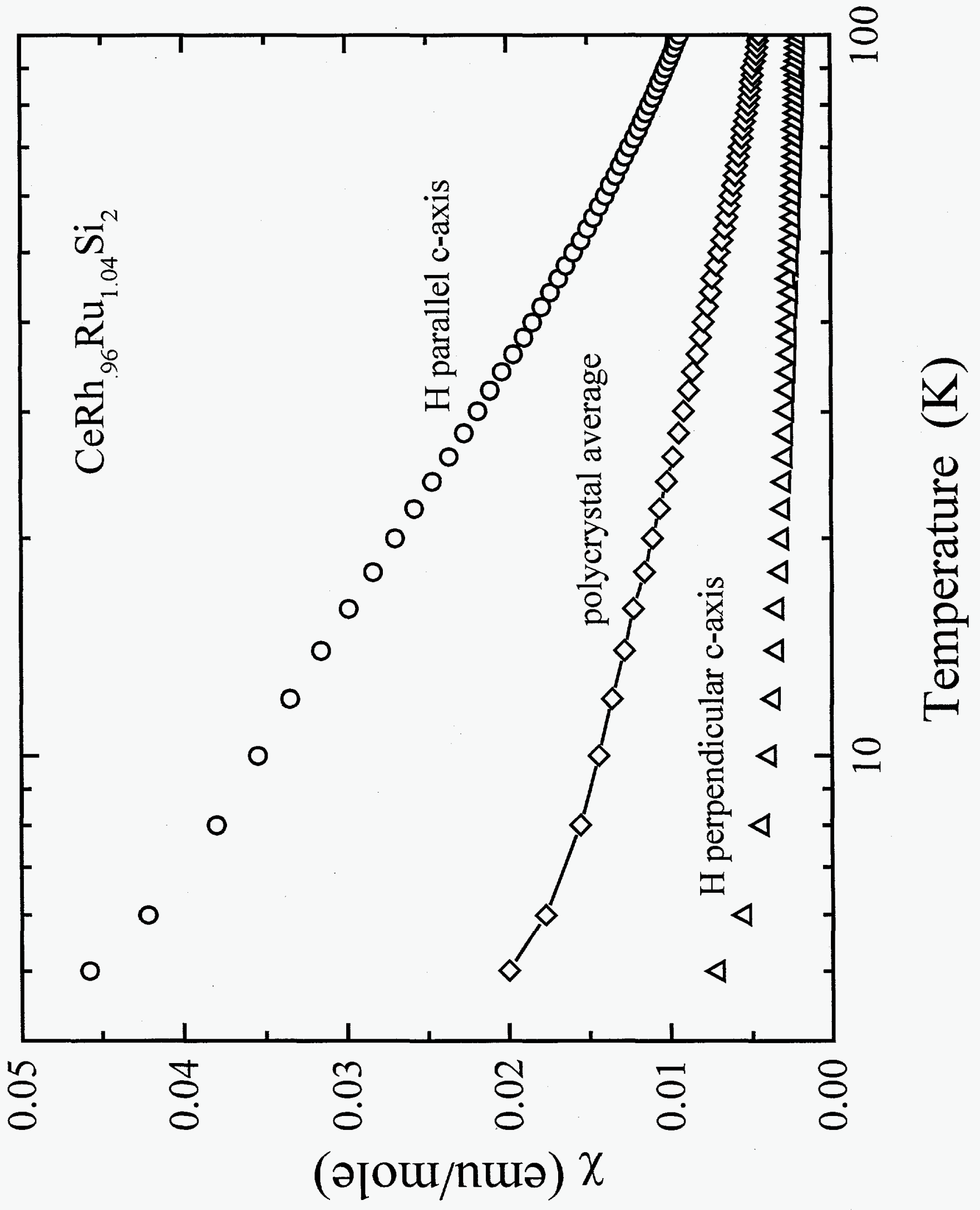

